\section{A COMPARISON OF COLONISING ORGANISMS WITH THOSE CAUSING ACQUIRED NEONATAL SEPTICAEMIA}

\author{
A. Stewart ${ }^{1}$, G. Inglis ${ }^{2,3}$ \\ ${ }^{1}$ Neonatology, Royal Hospital for Sick Children, \\ Glasgow, UK, ${ }^{2}$ Neonatology, Royal Brisbane and \\ Women's Hospital, '3epartment of Child Health, \\ The University of Queensland, Brisbane, QLD, \\ Australia
}

Aims: To compare organisms colonising infants in a tertiary level neonatal unit with those subsequently causing acquired nosocomial septicaemia.

Methods: We performed a retrospective audit of cases of culture-positive septicaemia in the Grantley Stable Neonatal Unit (GSNU) between January 2005 and December 2009. Cases were identified using the unit's database. We excluded those with onset of septicaemia before admission to the unit. Relevant data were extracted from the unit database and from patients' pathology reports. Specimens analysed included gastric aspirates, surface swabs, ETT aspirates, catheter tip cultures, and other clinically indicated swabs or specimens.

Results: During the study period there were 7572 admissions, with 116 episodes of septicaemia, acquired in the GSNU, in 105 patients. Two episodes involved co-infection with two organisms. The most common organisms causing septicaemia were enteric Gram-negative bacilli $(n=33)$, coagulasenegative Staphylococcus (CONS) species $(n=30)$ and Staphylococcus aureus $(n=23)$. Colonising organisms isolated most commonly were 'normal respiratory flora' not further specified (62 patients) and coagulase-negative staphylococci (44 patients). Septicaemia-causing and prior colonising organisms matched in 39/118 episodes (33\%). Another 17 (14\%) had possible matches (eg, Staphylococcus aureus versus 'mixed skin flora'). In 47 episodes $(40 \%)$ the organisms did not correlate. The remainder had no colonising organisms identified. Septicaemia-causing organisms were most likely to have been previously isolated from ETT aspirates $(n=17)$, post-admission surface swabs $(n=9)$, and umbilical catheter tips $(n=8)$.

Conclusions: There is relatively little correlation between acquired septicaemia and prior colonising organisms. Non-targeted surveillance may be of little clinical usefulness. Well-designed prospective studies are needed.

\section{NOSOCOMIAL INFECTION AND CD4+ LYMPHOCYTES IN VERY LOW BIRTH WEIGHT(VLBW) INFANTS'CORD BLOOD}

E. Zamora ${ }^{1}$, M. Sanchez Luna ${ }^{1}$, E. Maderueloํ․

S. Villar ${ }^{1}$, B. Alonso ${ }^{2}$, B. Padilla ${ }^{3}$, M. Cianchetta Sivori², S. Sánchez-Ramón ${ }^{2}$

${ }^{1}$ Neonatology, ${ }^{2}$ Immunology, ${ }^{3}$ Microbiology, HGU Gregorio Marañón, Madrid, Spain

Very low birth weight infants are at high risk for nosocomial infection specially due to higher survival rates related to advances in intensive care units. Prolonged hospital stay, instrumentalization and immaturity of their immune system are risk factors for infection, being gram-negative bacteria responsible for higher mortality rates and longterm sequelae. Patients and Methods: Prospective observational study in inborn VLBW infants, describing epidemiological features, morbidity and mortality in relation to risk factors sepsis and type of pathogen isolated in blood cultures. We perfomed flowcytomety analyses in cord blood samples and compared the lymphocyte subpopulations between the group of patients with nosocomial sepsis and the asymptomatic group. Results: 40 preterm infants were enrolled, mean birth weight (1059 \pm 197 grams) and 29,18 $\pm 2,05$ weeks postmenstrual age. $70 \%$ developed late-onset sepsis, with $65 \%$ coagulase-negative Stahylococcus, 31\% gramnegative bacteria and $4,4 \%$ Candida spp. Compared to those without sepsis, patent ductus arteriosus and bronchopulmonary dysplasia was significantly more frequent in the sepsis group $(91,7 \%$ vs $8,3 \%$; $p=0,02$; $85,7 \%$ vs $14,3 \% ; p=0,064)$, with longer duration of mechanical ventilation and $\mathrm{O}_{2}$ requirement $(3,40$ vs 15,04 days; $p=0,044 ; 16$ vs 46,96 days; $p=0,01$ ). CD4+ T lymphocytes were higher in umbilical cord blood in 28 VLBW neotates with sepsis ( 45,52+/12,13 vs $36,05+/-10,21 ; p=0,01$ ) but no differences were observed in CD8+ T cells. Conclusions: Umbilical cord blood phenotype of immune system differs in VLBW infants with nosocomial infection, with a higher percentage of CD4+ cells. The meaning of this finding and other possible differences remain to be fully interpreted. 